\title{
Impact of COVID-19 on maternal and perinatal outcomes in Harare, Zimbabwe: a comparative maternal audit
}

\author{
Yemurai Bikwa, Grant Murewanhema1, Mufaro Kanyangarara², Mugove G Madziyire', Zvavahera M Chirenje ${ }^{1}$ \\ 1 Unit of Obstetrics and Gynaecology, University of Zimbabwe, ${ }^{2}$ Department of Epidemiology and Biostatistics, Arnold School of Public Health, \\ University of South Carolina \\ Keywords: covid-19, maternal health, neonatal health, health services, zimbabwe
}

https://doi.org/10.29392/001c.28995

\section{Journal of Global Health Reports}

Vol. 5, 2021

\section{Background}

The COVID-19 pandemic lockdown resulted in the disruption of health services in Zimbabwe. The objectives of this study were two-fold. First, the study sought to determine the impact of the lockdown on maternal and perinatal outcomes at two tertiary hospitals in Harare, Zimbabwe, using a maternal audit. Second, the study sought to estimate the potential effect of COVID-19 related decreases in coverage of maternal and newborn health interventions on maternal, and neonatal mortality in Zimbabwe using the Lives Saved Tool (LiST).

\section{Methods}

A retrospective review of labour ward delivery registers, postnatal records and patients' maternity booklets was conducted. Data on total hospital deliveries, mode of delivery, and maternal and neonatal outcomes for March-August 2020 were abstracted and compared to data from March-August 2019. Logistic regression was performed to assess the association between maternal and perinatal outcomes and year as a proxy for the COVID-19 pandemic impact. Based on changes in coverage of interventions, LiST was used to estimate the impact of COVID-19 related disruptions on maternal and neonatal mortality at the national level.

\section{Results}

A total of 19,835 hospital deliveries that occurred at Sally Mugabe and Mbuya Nehanda Maternity Hospitals (11,346 for 2019 and 8,489 for 2020) were reviewed. Compared to 2019, there was a significant increased likelihood of breech deliveries (odds ratio, $\mathrm{OR}=1.2$; 95\% confidenxce interval, $\mathrm{CI}=1.1-1.5)$, uterine ruptures $(\mathrm{OR}=2.3 ; 95 \% \mathrm{CI}=1.1-5.1)$, stillbirths $(\mathrm{OR}=1.8 ; 95 \% \mathrm{CI}=1.5-2.2)$ and neonatal deaths $(\mathrm{OR}=1.4 ; 95 \% \mathrm{CI}=1.03-1.86)$ in 2020. However, there was a decrease in the odds of caesarean deliveries $(\mathrm{OR}=0.7 ; 95 \%$ $\mathrm{CI}=0.7-0.8$ ). From the LiST analysis, if similar disruptions to maternity services were observed across the country for a 12 month period, there would be an excess of 343 maternal deaths, 2,658 neonatal deaths and 450 stillbirths nationally.

\section{Conclusions}

These findings indicate a reduction in the utilisation of maternal health services and an increased risk of adverse maternal and neonatal outcomes during the nationwide lockdown. The provision of maternal and neonatal health services amid the pandemic remains crucial to reducing the negative indirect impacts of the pandemic.

The COVID-19 pandemic and resultant control measures brought unprecedented challenges to health service delivery across many countries. In Zimbabwe, a national lockdown was effected during the period March to August 2020 in the wake of the rising COVID-19 threat. This restricted public movement, prohibited gatherings and closed most services except those deemed essential. The provision of essential health services was disrupted, especially during the first wave. ${ }^{1}$ Maternity services were not spared, as an- tenatal, intrapartum and postnatal care services were disrupted. ${ }^{2}$ Movement restrictions and communication challenges brought about by the restrictive lockdowns added to the challenges faced by pregnant mothers in accessing healthcare services. ${ }^{3}$ During the lockdown, pregnant women were unable to access adequate antenatal care, failed to reach healthcare facilities on time, and faced delays in receiving care once at the hospital. ${ }^{2-8}$ While emergency procedures continued to take place, all elective surg- 
eries, including those for obstetric cases, were cancelled.

COVID-19 related disruptions in supply chains exacerbated pre-existing shortages of essential medicines and commodities for basic and comprehensive emergency obstetric care. ${ }^{5,8}$ In addition, shortages of personal protective equipment (PPE) for infection prevention and control led to the disruption of essential health services as health care workers feared contracting COVID-19.5,9,10 The increased risk of infection for health care workers, poor employee motivation and industrial actions by health care workers in the wake of the COVID-19 pandemic affected the availability of human resources for health. The COVID-19 pandemic also impacted the utilisation of health services, as evidenced by declines in outpatient attendances. ${ }^{1,7,11}$ A retrospective study at a tertiary hospital in Zimbabwe comparing routine maternal and perinatal statistics three months before and after the nationwide lockdown commenced found that the number of monthly hospital deliveries and caesarean section rates fell. In contrast, the number of women presenting in labour with unbooked pregnancies almost doubled. However, there was no significant change in maternal mortality, stillbirth rate, severe maternal morbidity such as post-partum haemorrhage ( $\mathrm{PPH})$, uterine ruptures, severe preeclampsia/eclampsia, and special care baby unit (SCBU) admissions.

Previous public health crises have provided valuable insights into maternal and perinatal outcomes. ${ }^{12,13}$ When the COVID-19 pandemic began, there was growing concern about the indirect effects of the COVID-19 pandemic on the provision and use of essential health services. ${ }^{14-16}$ Mathematical models were used to project the possible indirect consequences of the pandemic on health outcomes not directly linked to COVID-19 infection. ${ }^{16}$ The impact of the current COVID-19 pandemic on service provision and utilisation was expected to be similar but more wide-reaching than previous disease outbreaks such as Zika virus, SARS, and Ebola.

Understanding both the direct and indirect effects of pandemics on healthcare utilisation and health outcomes is crucial as it can help identify and minimise possible negative factors, help formulate policies and improve health outcomes. Several studies have assessed maternal and neonatal outcomes pre-COVID-19 and during the COVID-19 pandemic and have shown the detrimental effects on maternal and perinatal outcomes. ${ }^{14,16-19}$ For examples, studies in Ethiopia, Kenya, Malawi, and Uganda have reported significant increases in stillbirth rates, neonatal mortality, maternal mortality and decreases in institutional deliveries and quality of care provided. ${ }^{17,20-23}$ Therefore, objectives of this study were to: (1) determine the impact of the lockdown on maternal and perinatal outcomes at two tertiary hospitals in Harare, Zimbabwe, using a maternal audit, and (2) estimate the potential effect of COVID-19 related decreases in coverage of maternal and newborn health interventions on maternal, neonatal and stillbirth mortality at the national level using the Lives Saved Tool (LiST).

\section{METHODS}

\section{STUDY DESIGN AND SETTING}

This study was an analytical cross-sectional study of retrospective secondary data from two tertiary institutions that are affiliated with the University of Zimbabwe: Sally Mugabe Central Hospital and Mbuya Nehanda Maternity Hospital. Both hospitals receive referrals from surrounding municipal clinics and adjacent provinces and deliver a combined average of 25,000 babies per year. However, at the beginning of the COVID-19 pandemic in Zimbabwe, both hospitals substantially reduced health service provision to only life-threatening emergencies and stopped all nonemergency obstetric care.

Records of all obstetric patients receiving health care and neonates delivered or attended to at the two participating hospitals were assessed. Data on total hospital deliveries, modes of delivery, maternal and perinatal outcomes from March-August 2019 and March-August 2020 were abstracted from labour ward delivery registers, maternity theatre registers, postnatal records, and patients' maternity booklets, then checked for data quality. Outcomes of interest were total hospital deliveries, live births, stillbirths, vaginal deliveries, caesarean deliveries, breech deliveries, vacuum deliveries, uterine ruptures, peri-partum hysterectomies, maternal deaths, and neonatal deaths. Data from March-August 2019 was assumed to reflect hospitals operating under normal functional capacity before the pandemic. Data from March-August 2020 was assumed to reflect the impact of the nationwide lockdown, which was implemented to contain the first wave of the local outbreak.

\section{STATISTICAL ANALYSIS}

Ratios were calculated for maternal mortality, perinatal mortality, and caesarean section rates. Accidental and incidental causes of maternal death were excluded. Stillbirths were differentiated into intrapartum or fresh stillbirths (FSB) and macerated stillbirths (MSB). Frequencies and percentages were used for descriptive statistics for categorical variables. The year was considered the exposure of interest; 2019 was assumed to represent the period before the pandemic, whereas 2020 was the period during the pandemic. Univariate logistic regression models were used to assess the association between year and maternal/perinatal outcomes. Odds ratios and $95 \%$ confidence intervals were presented. A p value of less than 0.05 was considered statistically significant. All statistical analyses were performed using STATA version 16 (StataCorp: College Station, Texas).

The Lives Saved Tool (LiST) was used to demonstrate the potential impact of COVID-19 related disruptions in maternal and newborn health services on maternal, neonatal and stillbirth deaths at the national level. The LiST is a linear, deterministic model that estimates the impact of increases or decrease in intervention coverage on maternal, neonatal and child mortality. ${ }^{24}$ For this analysis, LiST was used to estimate the additional lives lost due to COVID-19 related service delivery and utilisation disruptions. A baseline projection was created for Zimbabwe with the reference year of 2019. Intervention coverage data was obtained from the 2015 Demographic and Health Survey (ZDHS) and the 2019 
Multiple Indicator Cluster Survey (MICS), nationally representative surveys with national and subnational estimates of key maternal, neonatal and child health (MNCH) indicators. $^{25,26}$ To estimate the potential impact of COVID-19 service-related disruptions on maternal and neonatal health outcomes nationally, we assumed that levels of service interruption nationally would be similar to those that occurred in the two participating hospitals. From 2019 to 2020 , there was a $25 \%$ decrease in facility deliveries in the two participating hospitals. While we assumed there was a $25 \%$ decrease in health service utilisation but no changes to the quality of care. No additional modifications were applied, and the default data in LiST was used. All LiST analyses were conducted using LiST Online (https://list.spectrumweb.org/).

\section{ETHICS CONSIDERATIONS}

Ethical approval was sought and obtained from the hospital regulatory authorities as well as the national authority: Sally Mugabe Hospital Ethics Committee (HCHEC010920/ 41), the Joint Research Ethics Committee (JREC/189/2020) of the University of Zimbabwe College of Health Sciences and Parirenyatwa Group of Hospitals, and the Medical Research Council of Zimbabwe (MRCZ/B/2028).

\section{RESULTS}

A total of 11,346 hospital deliveries occurred from MarchAugust 2019 and 8,489 hospital deliveries from March-August 2020 at Sally Mugabe Central Hospital Maternity Unit and Mbuya Nehanda Maternity Hospital (Table 1). There was a decline in the total hospital deliveries attended to in March-August 2020 during the lockdown compared to the same period in 2019. The difference in the total number of hospital deliveries was most noticeable in March and April 2020, coinciding with the lockdown's start (Figure 1). However, in May 2020, which corresponded to the initial relaxation of the nationwide lockdown, there was an increase in hospital deliveries compared to other months in the same year. Declines were also observed in the number of live births, vaginal deliveries, caesarean deliveries, and vacuum deliveries in 2020 (Table 1). Of the total hospital deliveries in 2019, 11,107 were live births compared to 8,008 in 2020, representing a $27.9 \%$ decline in the number of live births. In addition, there was a $31.2 \%$ decrease in the number of caesarean sections, from 3,402 in 2019 to 2,339 in 2020. The number of caesarean sections was lowest in July and August 2020 (Figure 2). There was also a reduction in vacuum deliveries in 2020 compared to 2019, with the lowest number of vacuum deliveries in July and August 2020 (Table 1).

By contrast, there was an increase in stillbirths, breech deliveries, and uterine ruptures in 2020 compared to 2019

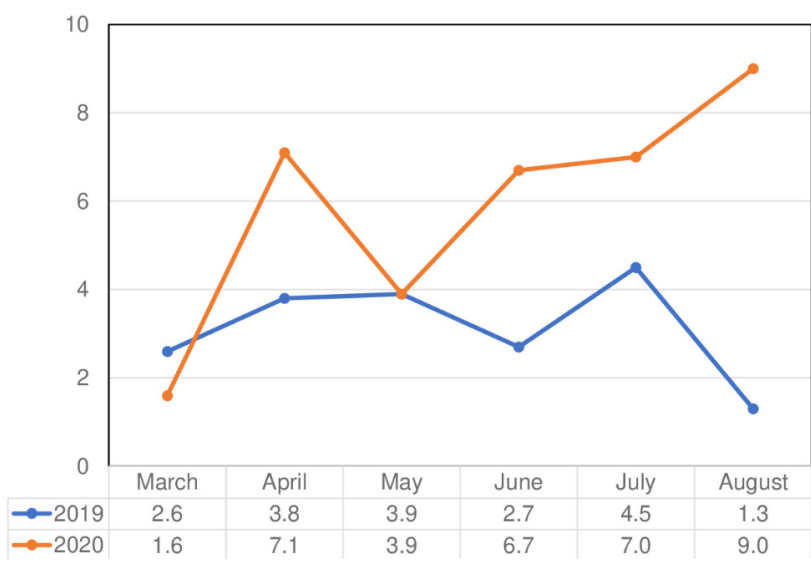

Figure 1. Maternal mortality ratio per 1000, MarchAugust 2019 and March-August 2020.

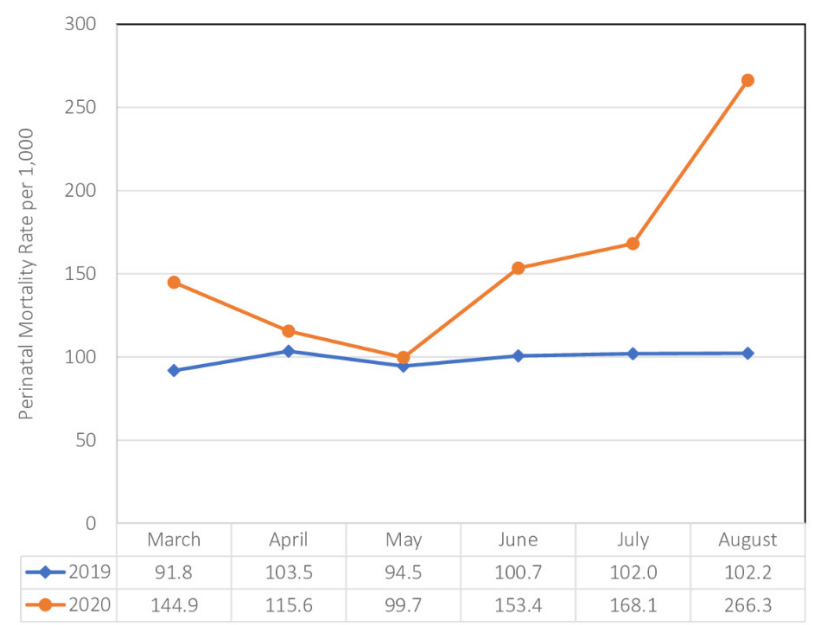

Figure 2. Perinatal mortality ratio per 1000, MarchAugust 2019 and March-August 2020.

(Table 1). The number of stillbirths increased by $21 \%$ from 508 in 2019 to 615 in 2020. This increase was primarily attributable to increased macerated stillbirths from 448 in 2019 to 558 in 2020 . There was little change in the number of fresh stillbirths with 60 in 2019 compared to 57 in 2020. There was a $33 \%$ increase in the number of breech deliveries in 2020 compared to 2019. The number of uterine ruptures doubled, from 10 in 2019 to 20 in 2020. There were 59\% fewer peripartum hysterectomies during the lockdown period compared to 2019, with April and August having no hysterectomies performed at all. 
Table 1. Trends in maternal and perinatal outcomes, March-August 2019 and March - August 2020.

\begin{tabular}{|c|c|c|c|c|c|c|c|c|c|c|c|c|c|c|}
\hline & \multicolumn{2}{|c|}{ March } & \multicolumn{2}{|c|}{ April } & \multicolumn{2}{|c|}{ May } & \multicolumn{2}{|c|}{ June } & \multicolumn{2}{|c|}{ July } & \multicolumn{2}{|c|}{ August } & \multicolumn{2}{|c|}{ Total } \\
\hline & 2019 & 2020 & 2019 & 2020 & 2019 & 2020 & 2019 & 2020 & 2019 & 2020 & 2019 & 2020 & 2019 & 2020 \\
\hline $\begin{array}{l}\text { Total hospital } \\
\text { deliveries }\end{array}$ & 1,947 & 1,345 & 1,895 & 967 & 2,098 & 2,665 & 1,851 & 1,431 & 2,033 & 1,215 & 1,522 & 866 & 11,346 & 8,489 \\
\hline $\begin{array}{l}\text { Vaginal } \\
\text { deliveries }\end{array}$ & $\begin{array}{r}1,337 \\
(68.7 \%)\end{array}$ & $\begin{array}{r}850 \\
(63.2 \%) \\
\end{array}$ & $\begin{array}{r}1303 \\
(68.8 \%) \\
\end{array}$ & $\begin{array}{r}809 \\
(70.1 \%)\end{array}$ & $\begin{array}{r}1485 \\
(70.8 \%)\end{array}$ & $\begin{array}{r}1812 \\
(68.0 \%) \\
\end{array}$ & $\begin{array}{r}1,286 \\
(69.5 \%)\end{array}$ & $\begin{array}{r}1,028 \\
(71.8 \%)\end{array}$ & $\begin{array}{r}1,392 \\
(68.4 \%)\end{array}$ & $\begin{array}{r}879 \\
(72.3 \%)\end{array}$ & $\begin{array}{r}934 \\
(61.4 \%)\end{array}$ & $\begin{array}{r}573 \\
(66.1 \%)\end{array}$ & $\begin{array}{r}7,737 \\
(68.1 \%)\end{array}$ & $\begin{array}{r}5,951 \\
(70.1 \%)\end{array}$ \\
\hline $\begin{array}{l}\text { Caesarean } \\
\text { deliveries }\end{array}$ & $\begin{array}{r}560 \\
(28.8 \%)\end{array}$ & $\begin{array}{r}379 \\
(28.2 \%)\end{array}$ & $\begin{array}{r}593 \\
(31.3 \%)\end{array}$ & $\begin{array}{r}345 \\
(29.9 \%)\end{array}$ & $\begin{array}{r}610 \\
(29.1 \%)\end{array}$ & $\begin{array}{r}733 \\
(27.5 \%)\end{array}$ & $\begin{array}{r}550 \\
(29.7 \%) \\
\end{array}$ & $\begin{array}{r}360 \\
(25.2 \%) \\
\end{array}$ & $\begin{array}{r}565 \\
(27.8 \%) \\
\end{array}$ & $\begin{array}{r}270 \\
(22.2 \%)\end{array}$ & $\begin{array}{r}524 \\
(34.4 \%)\end{array}$ & $\begin{array}{r}252 \\
(29.0 \%)\end{array}$ & $\begin{array}{r}3,402 \\
(30.0 \%)\end{array}$ & $\begin{array}{r}2,339 \\
(27.6 \%)\end{array}$ \\
\hline $\begin{array}{l}\text { Breech } \\
\text { deliveries }\end{array}$ & $\begin{array}{r}54 \\
(2.8 \%) \\
\end{array}$ & $\begin{array}{r}55 \\
(4.1 \%) \\
\end{array}$ & $37(2 \%)$ & $\begin{array}{r}49 \\
(5.1 \%) \\
\end{array}$ & $\begin{array}{r}34 \\
(1.6 \%) \\
\end{array}$ & $\begin{array}{r}106 \\
(4.0 \%) \\
\end{array}$ & $\begin{array}{r}38 \\
(2.1 \%) \\
\end{array}$ & $\begin{array}{r}51 \\
(3.6 \%) \\
\end{array}$ & $\begin{array}{r}55 \\
(2.7 \%) \\
\end{array}$ & $\begin{array}{r}43 \\
(3.5 \%) \\
\end{array}$ & $\begin{array}{r}32 \\
(2.1 \%) \\
\end{array}$ & $\begin{array}{r}28 \\
(3.2 \%) \\
\end{array}$ & $\begin{array}{r}250 \\
(2.2 \%) \\
\end{array}$ & $\begin{array}{r}332 \\
(3.9 \%) \\
\end{array}$ \\
\hline $\begin{array}{l}\text { Vacuum } \\
\text { deliveries }\end{array}$ & $\begin{array}{r}25 \\
(1.3 \%) \\
\end{array}$ & $\begin{array}{r}16 \\
(1.2 \%)\end{array}$ & $\begin{array}{r}15 \\
(0.8 \%) \\
\end{array}$ & $\begin{array}{r}16 \\
(1.7 \%)\end{array}$ & $\begin{array}{r}25 \\
(1.2 \%)\end{array}$ & $\begin{array}{r}33 \\
(1.2 \%)\end{array}$ & $\begin{array}{r}25 \\
(1.4 \%)\end{array}$ & $\begin{array}{r}15 \\
(1.0 \%)\end{array}$ & $\begin{array}{r}20 \\
(1.0 \%)\end{array}$ & $\begin{array}{r}5 \\
(0.4 \%)\end{array}$ & $\begin{array}{r}15 \\
(1.0 \%)\end{array}$ & $\begin{array}{r}3 \\
(0.3 \%)\end{array}$ & $\begin{array}{r}125 \\
(1.1 \%)\end{array}$ & $\begin{array}{r}88 \\
(1.0 \%) \\
\end{array}$ \\
\hline Live births & $\begin{array}{r}1,917 \\
(98.5 \%)\end{array}$ & $\begin{array}{r}1,277 \\
(94.9 \%)\end{array}$ & $\begin{array}{r}1,835 \\
(96.8 \%)\end{array}$ & $\begin{array}{r}986 \\
(92.6 \%)\end{array}$ & $\begin{array}{r}2,031 \\
(95.8 \%)\end{array}$ & $\begin{array}{r}2,587 \\
(93.9 \%)\end{array}$ & $\begin{array}{r}1,837 \\
(96.2 \%)\end{array}$ & $\begin{array}{r}1,349 \\
(92.8 \%)\end{array}$ & $\begin{array}{r}1,990 \\
(95.7 \%)\end{array}$ & $\begin{array}{r}1,148 \\
(91.9 \%)\end{array}$ & $\begin{array}{r}1,497 \\
(95.7 \%)\end{array}$ & $\begin{array}{r}661 \\
(90.5 \%)\end{array}$ & $\begin{array}{l}11,107 \\
(95.6 \%)\end{array}$ & $\begin{array}{r}8,008 \\
(92.9 \%)\end{array}$ \\
\hline Stillbirths & $91(4.7 \%)$ & $\begin{array}{r}95 \\
(7.1 \%) \\
\end{array}$ & $\begin{array}{r}98 \\
(5.2 \%) \\
\end{array}$ & $\begin{array}{r}79 \\
(7.4 \%) \\
\end{array}$ & $\begin{array}{r}90 \\
(4.2 \%) \\
\end{array}$ & $\begin{array}{r}167 \\
(6.0 \%) \\
\end{array}$ & $\begin{array}{r}72 \\
(3.8 \%) \\
\end{array}$ & $\begin{array}{r}104 \\
(7.2 \%) \\
\end{array}$ & $\begin{array}{r}90 \\
(4.3 \%) \\
\end{array}$ & $\begin{array}{r}101 \\
(8.1 \%) \\
\end{array}$ & $\begin{array}{r}67 \\
(4.3 \%) \\
\end{array}$ & $\begin{array}{r}69 \\
(9.5 \%) \\
\end{array}$ & $\begin{array}{r}508 \\
(4.4 \%) \\
\end{array}$ & $\begin{array}{r}615 \\
(7.1 \%) \\
\end{array}$ \\
\hline Fresh stillbirths & $\begin{array}{r}13 \\
(0.7 \%) \\
\end{array}$ & $\begin{array}{r}10 \\
(0.7 \%) \\
\end{array}$ & $\begin{array}{r}13 \\
(0.7 \%) \\
\end{array}$ & $\begin{array}{r}7 \\
(0.7 \%) \\
\end{array}$ & $\begin{array}{r}13 \\
(0.6 \%) \\
\end{array}$ & $\begin{array}{r}5 \\
(0.2 \%) \\
\end{array}$ & $\begin{array}{r}7 \\
(0.4 \%)\end{array}$ & $\begin{array}{r}10 \\
(0.7 \%) \\
\end{array}$ & $\begin{array}{r}9 \\
(0.4 \%)\end{array}$ & $\begin{array}{r}18 \\
(1.4 \%) \\
\end{array}$ & $\begin{array}{r}5 \\
(0.3 \%) \\
\end{array}$ & $\begin{array}{r}7 \\
(1.0 \%) \\
\end{array}$ & $\begin{array}{r}60 \\
(0.5 \%) \\
\end{array}$ & $\begin{array}{r}57 \\
(0.7 \%) \\
\end{array}$ \\
\hline $\begin{array}{l}\text { Macerated } \\
\text { stillbirths }\end{array}$ & $78(4 \%)$ & $\begin{array}{r}85 \\
(6.3 \%) \\
\end{array}$ & $\begin{array}{r}85 \\
(4.5 \%) \\
\end{array}$ & $\begin{array}{r}72 \\
(6.8 \%) \\
\end{array}$ & $\begin{array}{r}77 \\
(3.6 \%) \\
\end{array}$ & $\begin{array}{r}162 \\
(5.9 \%) \\
\end{array}$ & $\begin{array}{r}65 \\
(3.4 \%) \\
\end{array}$ & $\begin{array}{r}94 \\
(6.5 \%) \\
\end{array}$ & $\begin{array}{r}81 \\
(3.9 \%) \\
\end{array}$ & $\begin{array}{r}83 \\
(6.6 \%) \\
\end{array}$ & $\begin{array}{r}62 \\
(4.0 \%) \\
\end{array}$ & $\begin{array}{r}62 \\
(8.5 \%) \\
\end{array}$ & $\begin{array}{r}448 \\
(3.9 \%) \\
\end{array}$ & $\begin{array}{r}558 \\
(6.5 \%) \\
\end{array}$ \\
\hline $\begin{array}{l}\text { Uterine } \\
\text { ruptures }\end{array}$ & $2(0.1 \%)$ & $\begin{array}{r}4 \\
(0.3 \%) \\
\end{array}$ & $\begin{array}{r}1 \\
(0.05 \%) \\
\end{array}$ & $\begin{array}{r}5 \\
(0.5 \%) \\
\end{array}$ & $\begin{array}{r}1 \\
(0.05 \%) \\
\end{array}$ & $\begin{array}{r}4 \\
(0.2 \%) \\
\end{array}$ & $\begin{array}{r}2 \\
(0.1 \%) \\
\end{array}$ & $\begin{array}{r}2 \\
(0.1 \%) \\
\end{array}$ & $\begin{array}{r}2 \\
(0.1 \%) \\
\end{array}$ & $\begin{array}{r}1 \\
(0.1 \%) \\
\end{array}$ & $\begin{array}{r}2 \\
(0.1 \%) \\
\end{array}$ & $\begin{array}{r}4 \\
(0.5 \%) \\
\end{array}$ & $\begin{array}{r}10 \\
(0.1 \%) \\
\end{array}$ & $\begin{array}{r}20 \\
(0.2 \%) \\
\end{array}$ \\
\hline Hysterectomies & $2(0.1 \%)$ & $\begin{array}{r}5 \\
(0.4 \%) \\
\end{array}$ & $\begin{array}{r}3 \\
(0.2 \%) \\
\end{array}$ & $\begin{array}{r}0 \\
(0.0 \%) \\
\end{array}$ & $\begin{array}{r}3 \\
(0.1 \%) \\
\end{array}$ & $\begin{array}{r}4 \\
(0.2 \%) \\
\end{array}$ & $\begin{array}{r}3 \\
(0.2 \%) \\
\end{array}$ & $\begin{array}{r}7 \\
(0.5 \%) \\
\end{array}$ & $\begin{array}{r}7 \\
(0.3 \%) \\
\end{array}$ & $\begin{array}{r}3 \\
(0.2 \%) \\
\end{array}$ & $\begin{array}{r}4 \\
(0.3 \%) \\
\end{array}$ & $\begin{array}{r}0 \\
(0.0 \%) \\
\end{array}$ & $\begin{array}{r}22 \\
(0.2 \%) \\
\end{array}$ & $\begin{array}{r}13 \\
(0.2 \%) \\
\end{array}$ \\
\hline $\begin{array}{l}\text { Neonatal } \\
\text { deaths }\end{array}$ & $\begin{array}{r}96 \\
(4.9 \%)\end{array}$ & $\begin{array}{r}107 \\
(7.9 \%)\end{array}$ & $\begin{array}{r}103 \\
(5.4 \%)\end{array}$ & $\begin{array}{r}89 \\
(8.4 \%) \\
\end{array}$ & $\begin{array}{r}116 \\
(5.5 \%)\end{array}$ & $\begin{array}{r}95 \\
(3.4 \%)\end{array}$ & $\begin{array}{r}135 \\
(7.1 \%)\end{array}$ & $\begin{array}{r}108 \\
(7.4 \%)\end{array}$ & $\begin{array}{r}130 \\
(6.25 \%) \\
\end{array}$ & $\begin{array}{r}99 \\
(7.9 \%)\end{array}$ & $\begin{array}{r}99 \\
(6.3 \%)\end{array}$ & $\begin{array}{r}110 \\
(15.1 \%)\end{array}$ & $\begin{array}{r}679 \\
(5.8 \%)\end{array}$ & $\begin{array}{r}608 \\
(7.1 \%)\end{array}$ \\
\hline $\begin{array}{l}\text { Early neonatal } \\
\text { deaths }\end{array}$ & $\begin{array}{r}85 \\
(4.4 \%) \\
\end{array}$ & $\begin{array}{r}90 \\
(6.7 \%)\end{array}$ & $\begin{array}{r}92 \\
(4.9 \%)\end{array}$ & $\begin{array}{r}35 \\
(3.3 \%)\end{array}$ & $\begin{array}{r}102 \\
(4.8 \%)\end{array}$ & $\begin{array}{r}91 \\
(3.3 \%)\end{array}$ & $\begin{array}{r}113 \\
(5.9 \%)\end{array}$ & $\begin{array}{r}103 \\
(7.1 \%)\end{array}$ & $\begin{array}{r}113 \\
(5.4 \%)\end{array}$ & $\begin{array}{r}92 \\
(7.4 \%)\end{array}$ & $\begin{array}{r}86 \\
(5.5 \%)\end{array}$ & $\begin{array}{r}107 \\
(14.7 \%)\end{array}$ & $\begin{array}{r}591 \\
(5.1 \%)\end{array}$ & $\begin{array}{r}518 \\
(6.0 \%)\end{array}$ \\
\hline $\begin{array}{l}\text { Late neonatal } \\
\text { deaths }\end{array}$ & $\begin{array}{r}11 \\
(0.6 \%) \\
\end{array}$ & $\begin{array}{r}8 \\
(0.6 \%) \\
\end{array}$ & $\begin{array}{r}11 \\
(0.6 \%) \\
\end{array}$ & $\begin{array}{r}54 \\
(5.1 \%) \\
\end{array}$ & $\begin{array}{r}14 \\
(0.7 \%) \\
\end{array}$ & $\begin{array}{r}4 \\
(0.1 \%) \\
\end{array}$ & $\begin{array}{r}22 \\
(1.2 \%) \\
\end{array}$ & $\begin{array}{r}5 \\
(0.3 \%) \\
\end{array}$ & $\begin{array}{r}17 \\
(0.8 \%) \\
\end{array}$ & $\begin{array}{r}7 \\
(0.6 \%) \\
\end{array}$ & $\begin{array}{r}13 \\
(0.8 \%) \\
\end{array}$ & $\begin{array}{r}3 \\
(0.4 \%) \\
\end{array}$ & $\begin{array}{r}88 \\
(0.8 \%) \\
\end{array}$ & $\begin{array}{r}81 \\
(0.9 \%) \\
\end{array}$ \\
\hline $\begin{array}{l}\text { Maternal } \\
\text { deaths }\end{array}$ & $5(0.3 \%)$ & $\begin{array}{r}2 \\
(0.2 \%)\end{array}$ & $\begin{array}{r}7 \\
(0.4 \%)\end{array}$ & $\begin{array}{r}7 \\
(0.7 \%)\end{array}$ & $\begin{array}{r}8 \\
(0.3 \%)\end{array}$ & $\begin{array}{r}10 \\
(0.4 \%)\end{array}$ & $\begin{array}{r}5 \\
(0.3 \%)\end{array}$ & $\begin{array}{r}9 \\
(0.6 \%)\end{array}$ & $\begin{array}{r}9 \\
(0.4 \%)\end{array}$ & $\begin{array}{r}8 \\
(0.6 \%)\end{array}$ & $\begin{array}{r}2 \\
(0.1 \%)\end{array}$ & $\begin{array}{r}6 \\
(0.8 \%)\end{array}$ & $\begin{array}{r}36 \\
(0.3 \%)\end{array}$ & $\begin{array}{r}42 \\
(0.5 \%)\end{array}$ \\
\hline
\end{tabular}


Overall, the number of maternal deaths in 2020 was higher than the preceding year, with 36 deaths in 2019 and 42 in 2020 (Table 1). May, June, and July 2020 had the highest maternal deaths; the combined total accounted for $64.2 \%$ of the maternal deaths from March-August 2020. The maternal mortality ratio was higher in 2020 than in 2019, though the difference was not statistically significant (Figure 1).

The absolute number of neonatal deaths was lower in $2020(n=608)$ compared to $2019(n=679)$ (Table 1). However, the percentage of neonatal deaths was higher in 2020 than in 2019 (7.2\% compared to 6.0\%). As a result, the perinatal mortality rate (PMR) was higher in 2020, with a PMR of 142 per 1,000 births compared to 2019, which had a PMR of 99 per 1,000 births (Figure 2). In addition, an increase was noted in PMR from May 2020 to August 2020.

For maternal outcomes, there were increased odds of vaginal delivery, breech delivery and uterine rupture during the lockdown in March-August 2020 compared to the same period in 2019 (Table 2). The odds of vaginal delivery were 1.2 times more in 2020 than in 2019 (odds ratio, $\mathrm{OR}=1.2$; $95 \%$ confidence interval, $\mathrm{CI}=1.1-1.3)$. The odds of a breech delivery were of a similar magnitude $(\mathrm{OR}=1.2 ; 95 \% \mathrm{CI}=1.1$ $1.5)$. The odds of a uterine rupture were 2.3 times more in 2020 than in 2019; this was a statistically significant association $(\mathrm{OR}=2.3$; $95 \% \mathrm{CI}=1.1-5.1)$. By contrast, there was a decreased odds of delivery by caesarean section. In 2020, pregnant women delivered were $30 \%$ less likely to deliver by caesarean section than in 2019 (OR=0.7; 95\% CI=0.7 0.8 ). The odds of maternal death and peripartum hysterectomy were not significantly different between the two periods $(\mathrm{OR}=2.1,95 \% \mathrm{CI}=0.7-2.3 ; \mathrm{OR}=2.2$, 95\% $\mathrm{CI}=0.6-13.1$, respectively). For perinatal outcomes, there were increased odds of stillbirth and neonatal death in 2020 compared to 2019 (Table 2). There was an 80\% higher likelihood of stillbirth (OR=1.8; 95\% CI=1.5 - 2.2) and a 40\% higher likelihood of neonatal death $(\mathrm{OR}=1.4,95 \% \mathrm{CI}-1.0$ - 1.9) in 2020 compared to 2019.

Figure 3 shows a comparative analysis of the two periods, noting the major changes resulting from the disruption of services. There was an overall decrease in total hospital deliveries, live births, vaginal deliveries and caesarean sections during the lockdown period. Conversely, an increase in breech deliveries and neonatal deaths was noted during the lockdown period. Maternal deaths and uterine ruptures were represented by very small figures signifying the rarity of such events. The absolute figure of early neonatal deaths was slightly reduced in 2020 compared to 2019 . Overall this figure shows a reduction in utilisation of services.

Results from the LiST analysis projected that if utilisation of maternal and neonatal health services in Zimbabwe fell by $25 \%$ for 12 months, there would be an excess of 343 maternal deaths, 2,658 neonatal deaths and 450 stillbirths nationally. For maternal deaths, decreases in the availability of basic and emergency obstetric care would contribute to most of the excess maternal deaths resulting in an increase in the maternal mortality ratio from 458 to 539 deaths per 100,000 live births. More than half (57\%) of the additional deaths would be due to infections (sepsis, pneumonia and tetanus), which is the leading causes of death during the first month of life. Over the 12 months, the

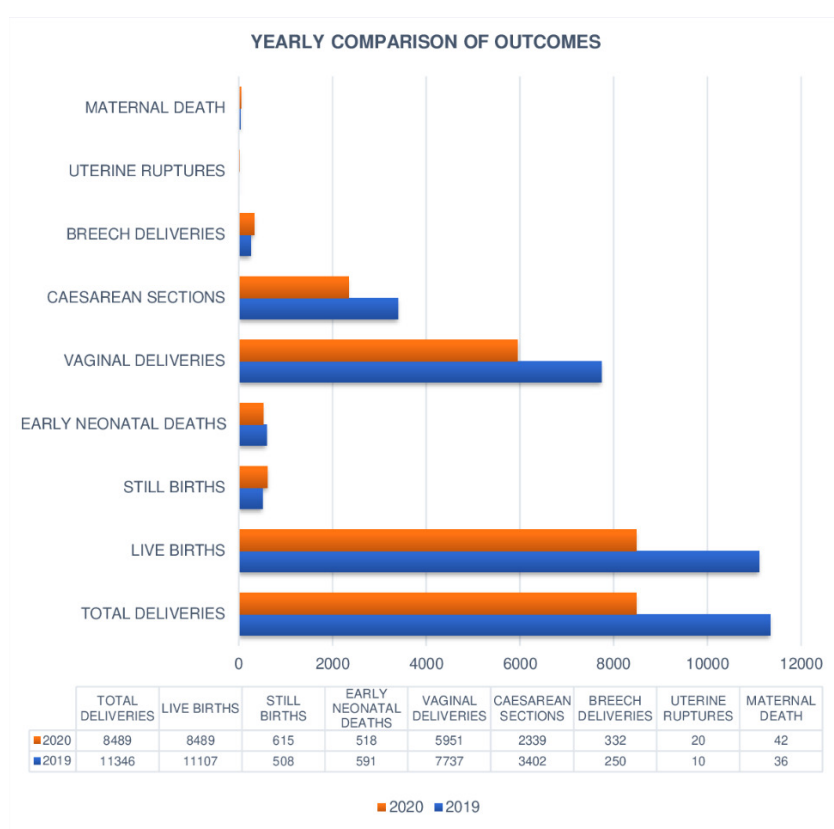

Figure 3. Yearly comparison of outcomes, 2019 versus 2020.

neonatal mortality rate would increase from 26 to 32 deaths per 1,000 live births. Notably, half (50\%) of the increase in stillbirths would result from decreased availability of cesarean delivery.

\section{DISCUSSION}

This study assessed the impact of the COVID-19 pandemic on maternal and perinatal outcomes in Harare, Zimbabwe, by comparing outcomes before the pandemic (March-August 2019) to during the pandemic (March-August 2020). Our findings suggest a decrease in utilisation of maternity services and an increase in adverse maternal and perinatal outcomes during the lockdown at Sally Mugabe Central Hospital Maternity Unit and Mbuya Nehanda Maternity Hospital. Additionally, if these increases were extrapolated to the entire country, there would an excess of 343 maternal deaths, 2,658 neonatal deaths and 450 stillbirths due to COVID-19 related disruptions to health service utilisation. There are several specific findings worth highlighting.

First, the study found that from March-August 2020, there was a reduction in the total number of hospital deliveries. This can be explained by reduced access to public transport, heavy enforcement of movement restrictions by the police and fear of infection among the general public. At the same time, service delivery during this period was also marred by health workers' fear of the threat posed by COVID-19, which was compounded by the increasing numbers of confirmed COVID-19 infections among health care workers, lack of PPE, and absence of safety precautions for infection prevention. ${ }^{2,4,9,10}$ Health workers also went on industrial action in protest of lack of sundries, PPE and commensurate remuneration. ${ }^{6}$ In response to the pandemic, tertiary level health facilities scaled-down operations. The scaling down of operations also took place at secondary and 
Table 2. Associations between maternal/perinatal outcomes and year from univariate logistic regression

\begin{tabular}{|c|c|c|c|c|}
\hline & $\begin{array}{c}\text { Year } 2019 \\
\text { n (\%) }\end{array}$ & $\begin{array}{c}\text { Year } 2020 \\
\mathrm{n}(\%)\end{array}$ & OR $(95 \% \mathrm{Cl})$ & $P$-value \\
\hline Vaginal delivery & & & & 0.004 \\
\hline No & $3,610(31.8)$ & $2,538(30)$ & Ref* & \\
\hline Yes & $7,736(68.2)$ & $5,951(70.1)$ & $1.2(1.1-1.3)$ & \\
\hline Caesarean delivery & & & & $<0.001$ \\
\hline No & $7,944(70.0)$ & $6,150(72.5)$ & Ref* $^{*}$ & \\
\hline Yes & $3,402(30.0)$ & $2,339(27.6)$ & $0.7(0.7-0.8)$ & \\
\hline Breech delivery & & & & $<0.001$ \\
\hline No & $11,096(97.8)$ & $8157(96.1)$ & Ref* & \\
\hline Yes & $250(2.2)$ & $332(3.9)$ & $1.2(1.1-1.5)$ & \\
\hline Vacuum delivery & & & & 0.66 \\
\hline No & 11,221 (98.9) & $8401(99)$ & Ref* $^{*}$ & \\
\hline Yes & $125(1.1)$ & $88(1)$ & $1.6(0.5-2.1)$ & \\
\hline Stillbirths & & & & $<0.001$ \\
\hline No & $10,838(95.5)$ & $7,874(92.8)$ & Ref* & \\
\hline Yes & $508(4.5)$ & $615(7.2)$ & $1.8(1.5-2.2)$ & \\
\hline Uterine rapture & & & & 0.008 \\
\hline No & $11,336(99.9)$ & $8,469(99.8)$ & $\operatorname{Ref}^{*}$ & \\
\hline Yes & $10(0.1)$ & $20(0.2)$ & $2.3(1.1-5.1)$ & \\
\hline Hysterectomy & & & & 0.5 \\
\hline No & 11,324 (99.8) & $8,476(99.9)$ & Ref* $^{*}$ & \\
\hline Yes & $22(0.12)$ & $13(0.2)$ & $2.2(0.6-13.1)$ & \\
\hline Neonatal deaths & & & & 0.001 \\
\hline No & $10,667(94)$ & 7,881 (92.8) & Ref* $^{*}$ & \\
\hline Yes & $679(6)$ & $608(7.2)$ & $1.4(1.0-1.9)$ & \\
\hline Early neonatal deaths & & & & 0.007 \\
\hline No & $10,755(94.8)$ & $7,971(94)$ & Ref* & \\
\hline Yes & $591(5.2)$ & $518(6.1)$ & $0.8(0.6-1.2)$ & \\
\hline Late neonatal deaths & & & & 0.2 \\
\hline No & $11,258(99.2)$ & 8,408 (99.1) & Ref* & \\
\hline Yes & $88(0.8)$ & $81(1)$ & $1.3(0.2-2.3)$ & \\
\hline Maternal death & & & & 0.2 \\
\hline No & 11,310 (99.7) & $8,453(99.6)$ & Ref* $^{*}$ & \\
\hline Yes & $36(0.3)$ & $42(0.4)$ & $2.1(0.8-2.3)$ & \\
\hline
\end{tabular}

*Ref-reference category $(\mathrm{OR}=1), \mathrm{P}=$ probability of the given outcome. Univariate logistic regression models were used to assess the association between year and maternal/perinatal outcomes

primary level health facilities, possibly contributing to the reduced number of referrals and subsequently reduced patient volumes. As concerning as the reduced service provision and patient volumes is the health status of missing obstetric patients, particularly those who did not seek care. It is possible that a proportion of the missing obstetric patients sought care through the private health sector. During the Ebola outbreak in Liberia, the number of deliveries in public government-owned health facilities dropped $27 \%$, whereas the number of deliveries in privately owned health facilities increased by $47 \%{ }^{13}$ In this situation, private health facilities created a cushion for the collapsed public health sector during the Ebola outbreak. However, given that less than $12 \%$ of Zimbabweans have medical insurance and costs of services and treatment is a major barrier to care, it is more likely that missing obstetric patients did not seek care and delivered at home. ${ }^{25}$ Based on the LiST analysis, this study also found that if the same declines in the utilisation of services were applied to the entire country, there would be excess stillbirths, neonatal and maternal deaths. While hypothetical, these results raise a concern about the magnitude of the impact of COVID-19 across the 
country, especially in more rural and remote areas.

Second, this study found a significant increase in stillbirths, particularly macerated stillbirths, during the lockdown. An increased number of stillbirths indicates poor antenatal or intrapartum care, as most stillbirths can be avoided with quality antenatal or intrapartum care. ${ }^{27}$ An increase in the number of fresh stillbirths indicates poor quality of intrapartum care, whereas an increase in the numbers of macerated stillbirths is a marker of poor antepartum care. ${ }^{27}$ During the lockdown, all out-patient clinics, including antenatal clinics at the two participating hospitals, were shut down, in a bid to promote social distancing and concentrate limited resources on the pandemic. The increase in stillbirths noted in this study may be attributable to a lack of antepartum monitoring as women had no access to antenatal clinics and auxiliary services such as ultrasound scan imaging and laboratory services.

Third, the study identified increased odds of vaginal delivery and breech delivery in 2020 compared to 2019. Possible explanations for the increased odds of vaginal delivery were most likely due to lack of theatre time caused by reduced staffing, reluctance to take patients into theatre because of lack of PPE, absence of standard operating protocols for infection prevention, poor health worker motivation and misinformation about the transmissibility of the virus. There were acute shortages in blood supply during the lockdown as regular blood donors were confined to their homes. This brought about reluctance in taking patients to theatre as blood products were in limited supply. In both China and Nepal, it was noted that women were more likely to deliver by caesarean section during the lockdown than before. ${ }^{18,28}$ However, it was noted in Wuhan that this was based on patients' requests. At the two participating hospitals in the present study, only one theatre is often available for elective obstetric cases, resulting in pressure for theatre time. Women offered caesarean delivery as personal preference end up delivering vaginally as emergencies take precedence. At the onset of the pandemic, elective theatre lists were cancelled, contributing to the increased likelihood of women delivering vaginally.

Breech deliveries are associated with poor foetal outcomes and have long term consequences on quality of life. ${ }^{29,30}$ The standard protocol at the participating hospitals is to deliver breech presentations in primups (first pregnancy) by caesarean section and carefully select those who can be allowed vaginal delivery. ${ }^{31}$ However, in 2020 the number of breech deliveries increased to 332 from 250 in 2019. This finding might reflect reduced theatre time, inadequate staffing, transportation problems leading to patients presenting in advanced labour and too late for caesarean delivery. An increase in breech deliveries may also be attributed to a lack of antenatal screening, as pregnant women with breech presentation typically will have elective caesarean deliveries. ${ }^{29-31}$

Fourth, the absolute number of caesarean sections and vacuum deliveries was lower than before the pandemic. Contributing factors may have been reduced overall number of deliveries, shortages of blood, staff shortages, and speculation fuelling fears among staff. The number of caesarean deliveries markedly declined during July and August 2020, coinciding with increasing SARS-COV-2 infection amongst health workers and the industrial action of middle-level doctors to improve PPE availability and advocate for the return of nurses to duty. While studies in other settings suggest an increase in the proportion of caesarean deliveries, this was not the case in the present study. ${ }^{23,28}$ A study on the impact of the lockdown on surgical cases in low and middle-income countries projected declines in surgical cases. ${ }^{32}$

Lastly, this study demonstrated a doubling in the likelihood of uterine ruptures in 2020 compared to 2019. The risk of uterine rupture is higher among women with previous uterine scars. ${ }^{33,34}$ Women with previous caesarean sections cannot receive antepartum care or get advance delivery plans with no antenatal clinic visits. Among women who received antenatal care in 2020, the quality of care was most likely substandard as there was no access to basic auxiliary services such as ultrasound imaging and laboratory services. Poor antepartum and intrapartum care most likely led to the poor perinatal outcomes observed in the present study. Contrary to our finding, another study conducted at Sally Mugabe Hospital neonatal intensive care unit (NICU) found that among that population, there was no change in neonatal outcomes from June 2019 to September 2020, suggesting that COVID-19 had not worsened neonatal outcomes for those admitted into NICU. 35

This study highlights the need to consider the indirect and direct impact of the COVID-19 response and mitigation measures on other health services. While Zimbabwe implemented strict and intense measures to flatten the curve and limit the spread of COVID-19, little effort was put into curbing the indirect effects of the pandemic. The increase in adverse maternal and perinatal outcomes documented in this study highlights the need to establish continuity of care even during the pandemic to provide essential services to vulnerable populations such as pregnant women and children. There is a need to strengthen the health system from the grassroots to decentralise care, which would be helpful during times when population movement is restricted. Health care workers also need support during pandemics as they are vulnerable to physical and mental breakdown and lack of motivation given increased exposure to COVID-19 in the health facility setting. Education of new conditions is always important, the lack of knowledge and standard guidelines can fuel fears and speculation resulting in inefficiencies in service delivery during the pandemic.

There are several limitations worth noting. First, this was a secondary analysis of retrospective data and could be subject to bias and errors at data collection. Second, as this data was collected for routine monthly maternal and neonatal statistical records at the relevant hospitals, there were limitations on the number of variables that could be assessed and potentially missing data and incomplete records. The present study identified associations, but no causal relationship could be established. More detailed information on demographics, possible confounders and causes of death would have added depth to the study; however, this information was unavailable from the data sources. Third, the findings may not represent the current service provision and maternal and perinatal outcomes as the study period of interest was March-August 2020. Additionally, the study only compared the data in March-August 2020 to that of 
March-August 2019, and it is possible that March-August 2019 may not represent typical secular trends in attendance and outcomes. Furthermore, this comparative audit was limited to two tertiary hospitals in Harare, which may limit the generalisability of the findings to lower-level facilities outside Harare. The inclusion of a more extended study period and more health facilities across all provinces of Zimbabwe would have strengthened the study and enhanced the generalisability of the findings. Lastly, for the LiST analysis, a $25 \%$ decrement was applied to the utilisation of maternity services and may not represent the actual changes. Additionally, the analysis focused on changes in the uptake of services and did not account for changes in the provision of services and quality of those services. Emerging evidence suggests the pandemic has also impacted the quality of available maternal and neonatal services. $^{21-23,25}$ While applying the $25 \%$ decrease in coverage was hypothetical, it represents the best available evidence given the paucity of coverage estimates during the pandemic.

\section{CONCLUSIONS}

Despite the limitations, the present study represents a comparative audit conducted at two tertiary hospitals in Harare that leveraged a record review of over 18,000 hospital deliveries during the study period. The indirect effects of COVID-19 on maternal and perinatal outcomes were observed. Although it is likely other factors may have been at play, the reduced caesarean section rate, increased perinatal mortality rate, increased number of stillbirths together with the increased likelihood of uterine ruptures and breech deliveries point towards disruptions in the provision of antepartum care and compromised quality of intrapartum care. As Zimbabwe continues to implement public health measures to curb the spread of COVID-19, strategies to reduce the indirect effects of the pandemic on maternal and perinatal outcomes are needed. Although the country is currently battling the third wave of the COVID-19 pandemic, these results are timely and useful for local, provincial, and national program planning.

\section{ACKNOWLEDGEMENTS}

We would like to thank the administrators at Sally Mugabe Central Hospital and Mbuya Nehanda Maternity Hospital for facilitating the conduct of this study and reproductive health officers at the two hospitals for compiling all the necessary data and assisting in the collation of missing data.

\section{FUNDING}

None.

\section{AUTHOR CONTRIBUTIONS}

YB, MGM, GM and ZMC conceptualised the study. YB collected the data. YB, and MK conducted the data analysis. YB and GM drafted the primary manuscript. MK, MGM, ZMC critically revised the manuscript for important intellectual content. All authors read and approved the final manuscript.

\section{COMPETING INTERESTS}

The authors completed the Unified Competing Interest form at http://www.icmje.org/disclosure-of-interest/ (available upon request from the corresponding author), and declare no conflicts of interest.

\section{CORRESPONDENCE TO:}

Dr Yemurai Bikwa, MD, MMed (Obstetrics and Gynaecology). Unit of Obstetrics and Gynaecology, Department of Primary Health Care Sciences, Faculty of Medicine and Health Sciences, University of Zimbabwe, PO Box A178, Avondale, Harare, Zimbabwe. Email: y.bikwa@yahoo.com.

Submitted: August 25, 2021 GMT, Accepted: September 20, 2021 GMT 


\section{REFERENCES}

1. Murewanhema G, Makurumidze R. Essential health services delivery in Zimbabwe during the COVID-19 pandemic: Perspectives and recommendations. Pan Afr Med J. 2020;35(Suppl 2). doi:10.11604/pamj.sup p.2020.35.2.25367

2. Murewanhema G, Nyakanda MI, Madziyire MG. Restoring and maintaining robust maternity services in the COVID-19 era: A public health dilemma in Zimbabwe. Pan Afr Med J. 2020;37(Suppl 1):32. doi:1 0.11604/pamj.supp.2020.37.1.26798

3. The devastating effects of COVID-19 on maternal health in Zimbabwe. Accessed August 11, 2021. http s://www.amnesty.org/en/latest/campaigns/2020/04/th e-devastating-effects-of-covid19-on-maternal-healt h-in-zimbabwe/

4. Harding A. Seven babies die in one night at Zimbabwe hospital. BBC News. Accessed August 11, 2021. https://www.bbc.com/news/world-africa-53580 559

5. Coronavirus: Zimbabwe doctors sue over "dire shortage” of protective gear. News24. Accessed August 9, 2021. https://www.news24.com/news24/afri $\mathrm{ca} /$ news/coronavirus-zimbabwe-doctors-sue-over-dir e-shortage-of-protective-gear-20200407

6. COVID-19 worsens Zimbabwe's health crisis. The Zimbabwean. Accessed March 26, 2021. https://www.t hezimbabwean.co/2020/08/covid-19-worsens-zimbab wes-health-crisis/

7. Shakespeare C, Dube H, Moyo S, Ngwenya S. Resilience and vulnerability of maternity services in Zimbabwe: a comparative analysis of the effect of Covid-19 and lockdown control measures on maternal and perinatal outcomes, a single-centre crosssectional study at Mpilo Central Hospital. BMC Pregnancy Childbirth. 2021;21(1):1-8. doi:10.1186/s12 884-021-03884-5

8. National Blood Transfusion Services Demand Advance Payments in Forex. News DzeZimbabwe. Accessed August 11, 2021. http://www.newsdzezimba bwe.co.uk/2020/07/national-blood-transfusion-servic es.html

9. Covid-19 update: 25 health workers test positive at Zimbabwean hospital. My Zimbabwe News. Accessed August 6, 2021. https://www.myzimbabwe.co.zw/new s/67653-covid-19-update-25-health-workers-test-pos itive-at-zimbabwean-hospital.html
10. More than 480 healthcare workers contract Covid-19. The Chronicle. Accessed August 11, 2021. $\underline{\mathrm{h}}$ ttps://www.chronicle.co.zw/more-than-480-healthcar e-workers-contract-covid-19/

11. Gavi S, Tapera O, Mberikunashe J, Kanyangarara M. Malaria incidence and mortality in Zimbabwe during the COVID-19 pandemic: analysis of routine surveillance data. Malar J. 2021;20(1):1-9. doi:10.118 6/s12936-021-03770-7

12. Sochas L, Channon AA, Nam S. Counting indirect crisis-related deaths in the context of a low-resilience health system: the case of maternal and neonatal health during the Ebola epidemic in Sierra Leone. Health Policy Plan. 2017;32(suppl_3):iii32-iii39. doi:1 0.1093/heapol/czx108

13. Gizelis TI, Karim S, Østby G, Urdal H. Maternal Health Care in the Time of Ebola: A Mixed-Method Exploration of the Impact of the Epidemic on Delivery Services in Monrovia. World Development. 2017;98:169-178. doi:10.1016/j.worlddev.2017.04.027

14. Ahmed SA, Ajisola M, Azeem K, et al. Impact of the societal response to COVID-19 on access to healthcare for non-COVID-19 health issues in slum communities of Bangladesh, Kenya, Nigeria and Pakistan: results of pre-COVID and COVID-19 lockdown stakeholder engagements. BMJ Glob Health. 2020;5(8):e003042. doi:10.1136/bmigh-2020-003042

15. World Health Organization. Critical preparedness, readiness and response actions for COVID-19-7. Published March 2020. Accessed August 19, 2021. htt ps://apps.who.int/iris/rest/bitstreams/1349213/retriev $\underline{\mathrm{e}}$

16. Roberton T, Carter ED, Chou VB, et al. Early estimates of the indirect effects of the COVID-19 pandemic on maternal and child mortality in lowincome and middle-income countries: a modelling study. The Lancet Global Health. 2020;8(7):e901-e908. doi:10.1016/s2214-109x(20)30229-1

17. Pallangyo E, Nakate MG, Maina R, Fleming V. The impact of covid-19 on midwives' practice in Kenya, Uganda and Tanzania: A reflective account. Midwifery. 2020;89:102775. doi:10.1016/j.midw.2020.102775

18. Ashish KC, Gurung R, Kinney MV, et al. Effect of the COVID-19 pandemic response on intrapartum care, stillbirth, and neonatal mortality outcomes in Nepal: a prospective observational study. The Lancet Global Health. 2020;8(10):e1273-81. doi:10.1016/s221 4-109x(20)30345-4 
19. Chmielewska B, Barratt I, Townsend R, et al. Effects of the COVID-19 pandemic on maternal and perinatal outcomes: a systematic review and metaanalysis. The Lancet Global Health.

2021;9(6):e759-e772. doi:10.1016/s2214-109x(21)000 $\underline{79-6}$

20. Kassie A, Wale A, Yismaw W. Impact of Coronavirus Diseases-2019 (COVID-19) on Utilization and Outcome of Reproductive, Maternal, and Newborn Health Services at Governmental Health Facilities in South West Ethiopia, 2020: Comparative Cross-Sectional Study. International Journal of Women's Health. 2021;13:479-488. doi:10.2147/ijwh.s $\underline{309096}$

21. Mzumara GW, Chawani M, Sakala M, et al. The health policy response to COVID-19 in Malawi. BMJ Glob Health. 2021;6(5):e006035. doi:10.1136/bmigh-2 021-006035

22. Burt JFM, Ouma J, Lubyayi L, et al. Indirect Effects of COVID-19 on Maternal, Neonatal, Child, Sexual and Reproductive Health Services in Kampala, Uganda. medRxiv. 2021.04.23.21255940. doi:10.1101/2 $\underline{021.04 .23 .21255940}$

23. Kimani RW, Maina R, Shumba C, Shaibu S. Maternal and newborn care during the COVID-19 pandemic in Kenya: Re-contextualising the community midwifery model. Hum Resour Health. 2020;18(1):75. doi:10.1186/s12960-020-00518-3

24. Walker N, Tam Y, Friberg IK. Overview of the Lives Saved Tool (LiST). BMC Public Health. 2013;13(Suppl 3):S1. doi:10.1186/1471-2458-13-s3-s1

25. Zimbabwe National Statistics Agency, ICF International. Zimbabwe Demographic and Health Survey 2015: Final Report. Zimbabwe National Statistics Agency (ZIMSTAT) and ICF International; 2016.

26. Zimbabwe National Statistics Agency (ZIMSTAT), UNICEF. Zimbabwe Multiple Indicator Cluster Survey 2019, Survey Findings Report. ZIMSTAT and UNICEF; 2019.

27. Lawn JE, Blencowe H, Pattinson R, et al. Stillbirths: Where? When? Why? How to make the data count? The Lancet. 2011;377(9775):1448-1463. d oi:10.1016/s0140-6736(10)62187-3
28. Li M, Yin H, Jin Z, et al. Impact of Wuhan lockdown on the indications of cesarean delivery and newborn weights during the epidemic period of COVID-19. PLOS ONE. 2020;15(8):e0237420. doi:10.13 71/journal.pone.0237420

29. Dohbit JS, Foumane P, Tochie JN, et al. Maternal and neonatal outcomes of vaginal breech delivery for singleton term pregnancies in a carefully selected Cameroonian population: a cohort study. BMJ Open. 2017;7(11):e017198. doi:10.1136/bmjopen-2017-0171 $\underline{98}$

30. RCOG statement on new Dutch study examining breech delivery and neonatal outcomes. Royal College of Obstetricians \& Gynaecologists. Accessed August 8, 2021. https://www.rcog.org.uk/en/news/rcog-state ment-on-new-dutch-study-examining-breech-deliver y-and-neonatal-outcomes/

31. Chirenje ZM. Harare - Essential Guide To Management of Common Obstetric and Gynaecologic. Scribd. Accessed August 17, 2021. https://www.scrib d.com/document/493046978/Harare-Essential-Guideto-Management-of-Common-Obstetric-and-Gynaeco $\underline{\operatorname{logic}}$

32. Mazingi D, Navarro S, Bobel MC, Dube A, Mbanje C, Lavy C. Exploring the Impact of COVID-19 on Progress Towards Achieving Global Surgery Goals. World J Surg. 2020;44(8):2451-2457. doi:10.1007/s002 68-020-05627-7

33. Hofmeyr GJ, Say L, Gülmezoglu AM. WHO systematic review of maternal mortality and morbidity: the prevalence of uterine rupture. BJOG. 2005;112(9):1221-1228. doi:10.1111/j.1471-0528.200 5.00725.x

34. Chiossi G, D’Amico R, Tramontano AL, Sampogna V, Laghi V, Facchinetti F. Prevalence of uterine rupture among women with one prior low transverse cesarean and women with unscarred uterus undergoing labor induction with PGE2: A systematic review and meta-analysis. PLOS ONE. 2021;16(7):e0253957. doi:10.1371/journal.pone.02539 $\underline{57}$

35. Chimhuya S, Neal SR, Chimhini G, et al. Indirect impacts of the COVID-19 pandemic at two tertiary neonatal units in Zimbabwe and Malawi: an interrupted time series analysis. medRxiv. 2021;2021.01.06.21249322. doi:10.1101/2021.01.06.2 1249322 